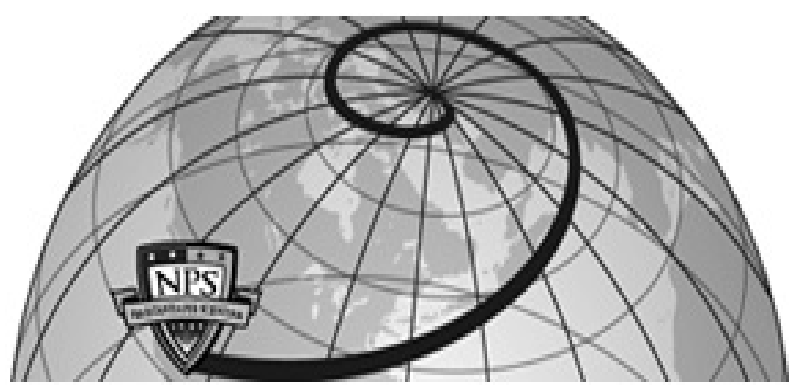

Calhoun: The NPS Institutional Archive DSpace Repository

\title{
Ionization potentials, experimental and theoretical, of the elements hydrogen to Krypton
}

Kelly, Raymond L.; Harrison, Don Edward

Monterey, California. Naval Postgraduate School

https://hdl.handle.net/10945/28971

This publication is a work of the U.S. Government as defined in Title 17, United States Code, Section 101. Copyright protection is not available for this work in the United States.

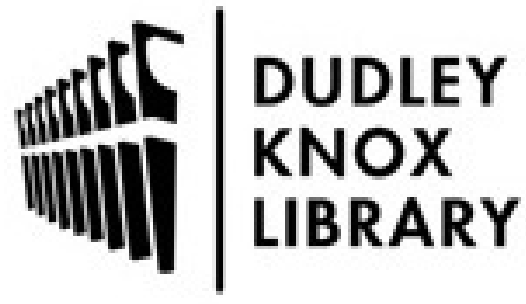

http://www.nps.edu/library
Calhoun is the Naval Postgraduate School's public access digital repository for research materials and institutional publications created by the NPS community. Calhoun is named for Professor of Mathematics Guy K. Calhoun, NPS's first appointed -- and published -- scholarly author.

Dudley Knox Library / Naval Postgraduate School 411 Dyer Road / 1 University Circle Monterey, California USA 93943 


\section{United States \\ Naval Postgraduate School}

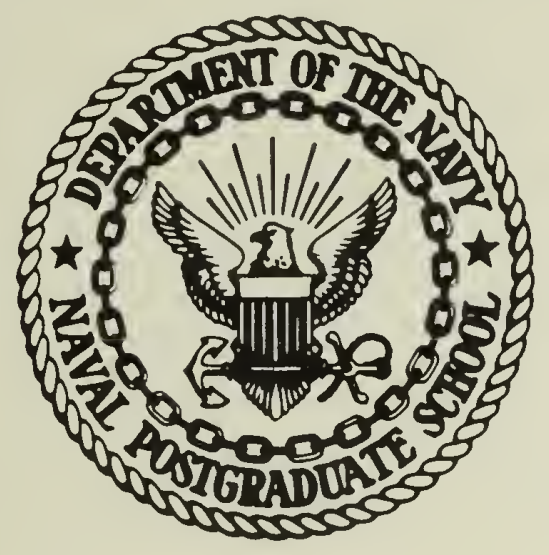

IONIZATION POTENTIALS, EXPERIMENTAL AND

THEORETICAL, OF THE ELEMENTS

HYDROGEN TO KRYPTON

by

Raymond I. Kelly and Don E. Harrison, Jr.

30 July 1970

This document has been approved for public release and sale; its distribution is unlimited. 

Rear Admiral R. W. McNitt, USN

Superintendent

R. F. Rinehart

Academic Dean

TITLE: Ionization Potentials, Experimental and Theoretical, of the Elements
Hydrogen to Krypton

\section{ABSTRACT:}

The ionization potentials of the isoelectronic sequences Helium through Zinc have been calculated by the Hartree-Fock method and compared with experimental results. Tables are presented showing the ionization potentials, as observed and extrapolated, for all stages of ionization of the first 36 elements.

This task was supported by: Naval Research Laboratory 
Because many of the ionization potentials of the lighter elements have not been measured, a numver of investigators ${ }^{1,2,3}$ have attempted to determine these potentials by interpolation and extrapolation from the available data. These efforts have generally taken the form of extrapolation along isoelectronic sequences through screening numuer analysis.

The most extensive recent compilation of ionization potentials is that of Lotz ${ }^{2}$, who presented results through the 26 th spectrum of $Z$ inc $(Z=30)$. His extrapolations were based on the expression

$$
T \approx \sum_{n=0}^{4} A_{n} \zeta^{n}+C(\zeta+B)^{-1}
$$

where $\zeta$ is the spectrum number. The $A_{n}, B$ and $C$ are constants within a sequence. Lotz fitted the experimental data to this formula, and he also included the type of relativistic correction suggested by Edlén ${ }^{1}$.

Here we undertake the same type of analysis, basing the extrapolation upon calculated approximations to the ionization potentials. The differences between the theoretical and experimental values are extrapolated, rather than the experimental data themselves. Because we extrapolate relatively small corrections to the theoretical values, the predicted ionization potentials should be quite reliable.

A number of computer programs now exist which will solve the radial portion of the Schrödinger equation for an atom or ion in the Hartree-Fock approximation. The most readily accessible of these are the Herman-Skillman ${ }^{4}$ (HS) and the Charlotte Froese Fischer (CFF) programs ${ }^{5}$. We have used these programs to compute ionization potentials for all states of ionization (except for the Hydrogen- 
like ions) for the elements from Helium to Zinc. The resulting values were compared against each other and against the experimental values. Where sufficient experimental data exist, the differences between calculated and experimental values were fitted to a second-order polynomial. This difference polynomial provides a reasonable extrapolation formula.

Any calculation of this type must inevitably involve a compromise between computer running time and the level of sophistication of the theoretical model. The Herman-Skillman program was chosen because it runs rapidly and requires a bare minimum of starting parameters. The resultant energy levels $E(H S)$ are the Hartree-Fock-Slater levels to the center of gravity of the ground state of the specified configuration. Ionization potentials calculated with this program usually deviate significantly from the experimental values, out the deviations are completely systematic along an isoelectronic sequence if the configuration does not change. Plausible extrapolations of the difference $\Delta_{\mathrm{HS}}=\mathrm{E}(\mathrm{HS})-\mathrm{E}(\mathrm{EXPT})$ can be made for all the systems studied.

The Charlotte Froese Fischer program is much more difficult to use. The stability of the numerical system is uncertain enough that the starting parameters must be adjusted by trial and error until self-consistent solutions finally result. However, because this program includes the electrostatic interactions between electrons through the Slater $F^{k}$ and $G^{k}$ integrals, the calculated ionization potentials $E(C F F)$ generally exhibit much better agreement with experiment than do the $E(H S)$ values. The extrapolated quantities $\Delta_{C F F}=E(C F F)-E(E X P T)$ are small, so these calculations can be used to predict ionization potentials with a confidence level of better than $\pm 0.3 \%$. 
The following tavles present the data in summary form, the experimental values veing derived from spectroscopic puolications ${ }^{2,6,7}$. In general, the criterion used in accepting numbers to fill the tables was that the empirically corrected values of the CFF and the HS calculations should agree. This criterion seems plausiole, since the two calculations require different corrections and the corrections are frequently of opposite sign.

In the ausence of a clear-cut relativistic contribution, the numvers given are vasically non-relativistic. For a few cases, Edlén ${ }^{1}$ indicates a possible relativistic correction. These have Deen included for the isoelectronic sequences of Helium, Lithium, Beryllium, Boron, Carbon and Sodium, as indicated in the comments below. 
H Sequence:

Precise calculations have been carried out by Garcia and Mack ${ }^{4}$ out to $Z=20$. Extrapolation to higher values of $Z$ was accomplished by fitting the calculated values to a fourth-order polynomial, leading to the following formula:

$$
T=\left(1.85097 \times 10^{-4}\right)(Z-0.41602)^{4}+13.6063 Z^{2}-\left(4.58298 \times 10^{-3}\right) Z-0.0032
$$

\section{Spectrum}

$\mathrm{H} \mathrm{I}$

He II

Li III

Be IV

B V

C VI

$\mathrm{N}$ VII

O VIII

F IX

$\mathrm{Ne} X$

Na XI

Mg XII

Al XIII

SI XIV

$P X V$

$S X V I$

Cl XVII

Ar XVIII

$\mathrm{K}$ XIX

Ca XX

Sc XXI

Ti XXII

V XXIII

Cr XXIV

Mn XXV

Fe XXVI

Co XXVII

Ni XXVIII

$\mathrm{Cu}$ XXIX

Zn XXX

Ga XXXI

Ge XXXII

As XXXIII

Se XXXIV

Br XXXV

Kr XXXVI

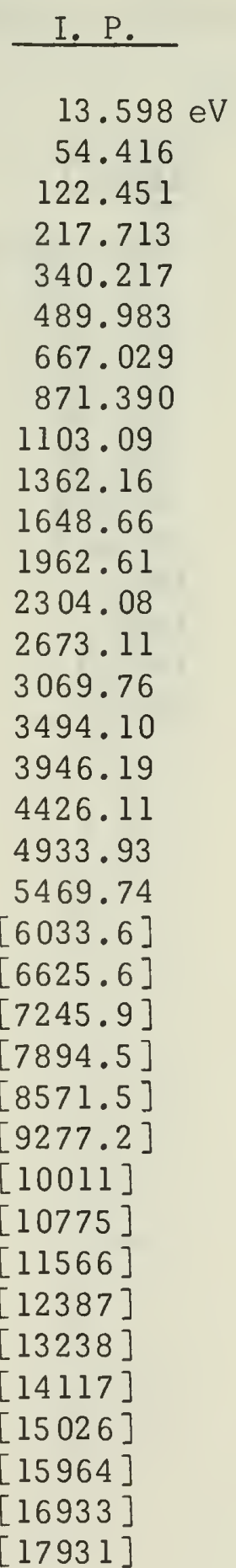


We used the relativistic correction proposed by Edlén ${ }^{1}$, $\Delta_{\mathrm{R}}=\left(1.8112 \times 10^{-4}\right) \zeta^{4}$ where the spectrum number $\zeta=\mathrm{Z}-1$. When this correction was subtracted from the experimental results, the resulting reduced values fit very well to $\mathrm{T}_{\mathrm{N}}=13.6002 \zeta^{2}+10.2436 \zeta+0.74314$ or to an Edlén type formula as used by Lotz. ${ }^{N}$ (The ionization potential of $\mathrm{H}^{-}$is thus calculated to be $0.743 \mathrm{eV}$, compared with the accurate value of 0.755. ) The CFF values are almost exactly the same as the reduced values $\mathrm{T}_{\mathrm{N}}$. The final formula is:

$$
T=\left(1.8112 \times 10^{-4}\right) \zeta^{4}+13.6002 \zeta^{2}+10.2436 \zeta+0.74314 .
$$

\begin{tabular}{|c|c|c|c|c|}
\hline Spectrum & $\mathrm{E}(\mathrm{EXPT})$ & $\begin{array}{l}\text { Uncorrected } \\
\mathrm{E}(\mathrm{CFF}) \\
\end{array}$ & $\Delta_{\mathrm{R}}$ & I. P. \\
\hline $\mathrm{He} \mathrm{I}$ & $24.587 \mathrm{eV}$ & $24.978 \mathrm{eV}$ & $\mathrm{eV}$ & $24.587 \mathrm{eV}$ \\
\hline Li II & 75.638 & 75.980 & 0.003 & 75.638 \\
\hline $\mathrm{Be}$ III & 153.89 & 154.202 & .015 & 153.89 \\
\hline B IV & 259.36 & 259.637 & .046 & 259.36 \\
\hline $\mathrm{C} \mathrm{V}$ & 392.08 & 392.283 & .113 & 392.08 \\
\hline$N V I$ & 552.06 & 552.141 & .235 & 552.06 \\
\hline O VII & 739.30 & 739.208 & .435 & 739.30 \\
\hline F VIII & 954.84 & 953.486 & .742 & 953.84 \\
\hline $\mathrm{Ne}$ IX & 1196 & 1194.97 & 1.19 & 1195.73 \\
\hline Na X & 1465 & 1463.67 & 1.81 & $146 \overline{.00}$ \\
\hline Mg XI & 1761.7 & 1759.58 & 2.65 & 1761.70 \\
\hline Al XII & 2086.0 & 2082.70 & 3.78 & $208 \underline{.85}$ \\
\hline Si XIII & & 2433.03 & 5.17 & {$[2438]$} \\
\hline P XIV & & 2810.57 & 6.96 & {$[2817]$} \\
\hline S XV & & 3215.31 & 9.17 & {$[3224]$} \\
\hline Cl XVI & & 3647.27 & 11.87 & {$[3658]$} \\
\hline Ar XVII & & 4106.44 & 15.13 & {$\left[\begin{array}{llll}4 & 12 & 0\end{array}\right]$} \\
\hline K XVIII & & 4592.82 & 19.01 & {$\left[\begin{array}{llll}4 & 6 & 1 & 1\end{array}\right]$} \\
\hline Ca XIX & & 5106.41 & 23.60 & {$\left[\begin{array}{lll}5 & 129\end{array}\right]$} \\
\hline Sc $X X$ & & 5647.21 & 28.98 & {$[5675]$} \\
\hline $\mathrm{Ti} X X I$ & & 6215.22 & 35.22 & {$[6249]$} \\
\hline V XXII & & 6810.43 & 42.43 & {$[6851]$} \\
\hline Cr XXIII & & 7432.86 & 50.68 & {$[7482]$} \\
\hline Mn XXIV & & 8082.50 & 60.09 & {$[8141]$} \\
\hline Fe XXV & & 8759.35 & 70.75 & {$[8828]$} \\
\hline Co XXVI & & 9463.41 & 82.77 & {$[9544]$} \\
\hline Ni XXVII & & 10194.7 & 96.3 & [1 0288$]$ \\
\hline Cu XXVIII & & 10953.2 & 111.3 & {$[11062]$} \\
\hline Zn XXIX & & 11738.8 & 128.1 & [11864] \\
\hline Ga XXX & & 1256.7 .7 & 146.7 & [12695] \\
\hline Ge XXXI & & 13391.8 & 167.3 & {$[13556]$} \\
\hline As XXXII & & 14259.2 & 189.9 & {$[14445]$} \\
\hline Se XXXIII & & 15153.7 & 214.8 & {$[15365]$} \\
\hline Br XXXIV & & 16075.4 & 242.0 & {$\left[\begin{array}{lll}163 & 13\end{array}\right]$} \\
\hline $\mathrm{Kr} X X X V$ & & 17024.4 & 271.8 & {$[17292]$} \\
\hline
\end{tabular}


Li Sequence:

The data fit well when the Edlén relativistic correction is used. The final formula is (with $\zeta=\mathrm{Z}-2$ ) :

\begin{tabular}{|c|c|c|c|c|c|}
\hline Spectrum & $\underline{E(E X P T)}$ & $\begin{array}{c}\text { Uncorrected } \\
\mathrm{E}(\mathrm{CFF}) \\
\end{array}$ & $\Delta_{\mathrm{R}}$ & & I. P. \\
\hline Li I & 5.390 & $\mathrm{~V} \quad 5.342 \mathrm{eV}$ & 0 & $\mathrm{eV}$ & $5.390 \mathrm{eV}$ \\
\hline $\mathrm{Be}$ II & 18.211 & 18.126 & 0.005 & & 18.211 \\
\hline B III & 37.930 & 37.818 & .014 & & 37.930 \\
\hline C IV & 64.492 & 64.352 & .035 & & 64.492 \\
\hline $\mathrm{N} \mathrm{V}$ & 97.888 & 97.707 & .073 & & 97.888 \\
\hline O VI & 138.115 & 137.876 & .136 & & 138.115 \\
\hline F VII & 185.185 & 184.854 & .232 & & 185.185 \\
\hline Ne VIII & 239.1 & 238.64 & .37 & & 239.10 \\
\hline $\mathrm{Na}$ IX & 299.85 & 299.23 & .57 & & 299.85 \\
\hline $\operatorname{Mg} X$ & 367.46 & 366.62 & .83 & & 367.46 \\
\hline Al XI & 442.0 & 440.82 & 1.17 & & 442.00 \\
\hline Si XII & 523.38 & 521.82 & 1.62 & & 523.38 \\
\hline P XIII & 611.60 & 609.63 & 2.17 & & 611.60 \\
\hline S XIV & $804.6 ?$ & 704.24 & 2.86 & & [707] \\
\hline $\mathrm{Cl} \mathrm{XV}$ & & 805.65 & 3.71 & & [809] \\
\hline Ar XVI & & 913.87 & 4.73 & & [918] \\
\hline K XVII & & 1028.89 & 5.94 & & [1034] \\
\hline Ca XVIII & & 1150.71 & 7.38 & & [ 1157$]$ \\
\hline Sc XIX & & 1279.33 & 9.06 & & [1288] \\
\hline Ti $X X$ & & 1414.76 & 11.01 & & [1425] \\
\hline V XXI & & 1556.99 & 13.26 & & {$[1569]$} \\
\hline Cr XXII & & 1706.02 & 15.84 & & [1720] \\
\hline Mm XXIII & & 1861.86 & 18.78 & & [1879] \\
\hline Fe XXIV & & 2024.50 & 22.11 & & [2 045$]$ \\
\hline Co XXV & & 2193.94 & 25.86 & & [22 218 ] \\
\hline $\mathrm{Ni} X X V I$ & & 2370.18 & 30.08 & & [2398] \\
\hline Cu XXVII & & 2553.22 & 34.79 & & [2585] \\
\hline Zn XXVIII & & 2743.07 & 40.03 & & [2780] \\
\hline Ga XXIX & & 2939.72 & 45.84 & & [2982] \\
\hline Ge $X X X$ & & 3143.18 & 52.27 & & [3 192 ] \\
\hline As XXXI & & 3353.43 & 59.35 & & [3409] \\
\hline Se XXXII & & 3570.49 & 67.12 & & [3633] \\
\hline Br XXXIII & & 3794.35 & 75.63 & & [3865] \\
\hline Kr XXXIV & & 4025.02 & 84.93 & & {$[4105]$} \\
\hline
\end{tabular}


The non-relativistic extrapolated values are the CFF energies with a $\zeta^{2}$ correction subtracted.

\section{Spectrum}

$\mathrm{Be} I$

B II

C III

$\mathrm{N}$ IV

O V

F VI

$\mathrm{Ne}$ VII

Na VIII

Mg IX

Al X

Si XI

$P$ XII

$X$ XIII

Cl XIV

Ar XV

$\mathrm{K} X V I$

Ca XVII

Sc XVIII

Ti XIX

$\mathrm{V} X \mathrm{X}$

Cr XXI

Mn XXII

Fe XXIII

Co XXIV

$\mathrm{Ni} X X V$

$\mathrm{Cu}$ XXVI

Zn XXVII

Ga XXVIII

Ge XXIX

As XXX

Se XXXI

Br XXXII

Kr XXXIII

$E(E X P T)$
\[ .322 \mathrm{eV} \]
95.155
47.883
77.471
113.896
157.156
207.3
264.22
327.98
398.6
476.15
560.5

560.5
Uncorrected

\begin{tabular}{c}
$E(C F F)$ \\
\hline $8.415 \mathrm{eV}$ \\
23.777 \\
46.095 \\
75.283 \\
111.309 \\
154.158 \\
203.82 \\
260.30 \\
323.58 \\
393.68 \\
470.58 \\
554.28 \\
644.79 \\
742.10 \\
846.22 \\
957.14 \\
1074.9 \\
1199.4 \\
1330.7 \\
1468.9 \\
1613.8 \\
1765.5 \\
1924.1 \\
2089.4 \\
2261.6 \\
2440.5 \\
2626.3 \\
2818.8 \\
3018.2 \\
3224.4 \\
3437.3 \\
3657.1 \\
3883.7 \\
\end{tabular}

I. P.

$9.322 \mathrm{eV}$

25.155

47.883

77.471

113.896

157.156

207.27

264.22

327.98

398.6

476.15

560.5

(652)

(749)

(854)

(966)

(1084)

(1209)

(1342)

(1480)

(1626)

(1779)

(1938)

(2104)

(2277)

(2457)

(2644)

(2837)

(3038)

(3245)

(3459)

(3680)

(3907) 
B Sequence:

The non-relativistic extrapolated values are the CFF energies as calculated.

\begin{tabular}{|c|c|c|}
\hline Spectrum & $\underline{E(E X P T)}$ & $\begin{array}{c}\text { Uncorrected } \\
\mathrm{E}(\mathrm{CFF}) \\
\end{array}$ \\
\hline B I & $8.298 \mathrm{eV}$ & $8.431 \mathrm{eV}$ \\
\hline C II & 24.382 & 24.618 \\
\hline$N$ III & 47.438 & 47.770 \\
\hline O IV & 77.412 & 77.815 \\
\hline F V & 114.24 & 114.72 \\
\hline $\mathrm{Ne} \mathrm{VI}$ & 157.95 & 158.45 \\
\hline $\mathrm{Na}$ VII & 208.50 & 209.02 \\
\hline Mg VIII & 266.02 & 266.40 \\
\hline Al IX & 330.2 & 330.60 \\
\hline Si X & 401.38 & 401.61 \\
\hline P XI & 479.5 & 479.44 \\
\hline S XII & & (564) \\
\hline Cl XIII & & (656) \\
\hline Ar XIV & & (754) \\
\hline $\mathrm{K} X \mathrm{XV}$ & & (859) \\
\hline Ca XVI & & (97 1) \\
\hline Sc XVII & & (1089) \\
\hline Ti XVIII & & (12 15) \\
\hline V XIX & & (1347) \\
\hline Cr XX & & (1486) \\
\hline $\mathrm{Mn} X X I$ & & (1632) \\
\hline Fe XXII & & (1784) \\
\hline Co XXIII & & (1944) \\
\hline $\mathrm{Ni} X X \mathrm{XV}$ & & (2110) \\
\hline $\mathrm{Cu} \mathrm{XXV}$ & & (2284) \\
\hline Zn XXVI & & (2463) \\
\hline Ga XXVII & & $(2650)$ \\
\hline Ge XXVIII & & $(2844)$ \\
\hline As XXIX & & $(3044)$ \\
\hline Se XXX & & (3251) \\
\hline $\operatorname{Br} X X X I$ & & $(3465)$ \\
\hline $\mathrm{Kr}$ XXXII & & (3686) \\
\hline
\end{tabular}




\section{Sequence:}

The non-relativistic extrapolated values are the CFF energies with a $\zeta^{2}$ correction subtracted.

Spectrum

C I

$\mathrm{N}$ II

O III

F IV

$\mathrm{Ne} \mathrm{V}$

Na VI

Mg VII

Al VIII

Si IX

$\mathrm{P} X$

$S$ XI

Cl XII

Ar XIII

$\mathrm{K}$ XIV

$\mathrm{Ca} X \mathrm{~V}$

Sc XVI

Ti XVII

V XVIII

Cr XIX

Mn XX

Fe XXI

Co XXII

Ni XXIII

$\mathrm{Cu}$ XXIV

Zn XXV

Ga XXVI

Ge XXVII

As XXVIII

Se XXIX

$\mathrm{Br} X X X$

Kr XXXI
$\underline{E(E X P T)}$

$11.260 \mathrm{eV}$

29.600

54.900

87.16

126.29

172.14

224.95

284.6

351.05

424.4
Uncorrected

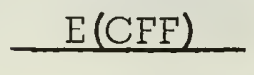

$11.791 \mathrm{eV}$

30.206

55.614

87.93

127.12

173.15

226.02

285.71

352.22

425.54

505.7

592.6

686.4

787.0

894.3

1009

1130

1257

1392

1533

1682

1837

1998

2167

2342

2525

2714

2909

3112

3322

3538

\begin{tabular}{l} 
I.P. \\
\hline $11.260 \mathrm{eV}$ \\
29.600 \\
54.900 \\
87.16 \\
126.29 \\
172.14 \\
224.95 \\
284.6 \\
351.05 \\
424.4 \\
$(504)$ \\
$(591)$ \\
$(685)$ \\
$(785)$ \\
$(893)$ \\
$(1007)$ \\
$(1128)$ \\
$(1255)$ \\
$(1390)$ \\
$(1531)$ \\
$(1679)$ \\
$(1834)$ \\
$(1996)$ \\
$(2165)$ \\
$(2340)$ \\
$(2522)$ \\
$(2711)$ \\
$(2907)$ \\
$(3109)$ \\
$(3319)$ \\
$(3535)$ \\
\end{tabular}




\section{N Sequence:}

The non-relativistic extrapolated values are the CFF energies with a $\zeta^{2}$ correction subtracted.

Spectrum

$\mathrm{N} \mathrm{I}$

O II

F III

Ne IV

$\mathrm{Na} \mathrm{V}$

Mg VI

Al VII

Si VIII

$P$ IX

$\mathrm{S} X$

Cl XI

Ar XII

$\mathrm{K}$ XIII

Ca XIV

Sc XV

Ti XVI

V XVII

Cr XVIII

Mn XIX

Fe XX

Co XXI

Ni XXII

$\mathrm{Cu}$ XXIII

Zn XXIV

Ga XXV

Ge XXVI

As XXVII

Se XXVIII

Br XXIX

$\mathrm{Kr} X X X$

$\underline{E(E X P T)}$
$14.532 \mathrm{eV}$
35.117
62.66
97.05
138.40
186.54
241.40
303.15
371.6
447.1
529.3
618.5

Uncorrected

$\mathrm{E}(\mathrm{CFF})$

$15.444 \mathrm{eV}$

36.105

63.776

98.37

139.84

188.16

243.33

305.32

374.14

449.78

532.24

621.5

717.6

820.5

930.2

1047

1170

1300

1437

1581

1731

1889

2053

2224

2401

2586

2777

2976

3181

3392

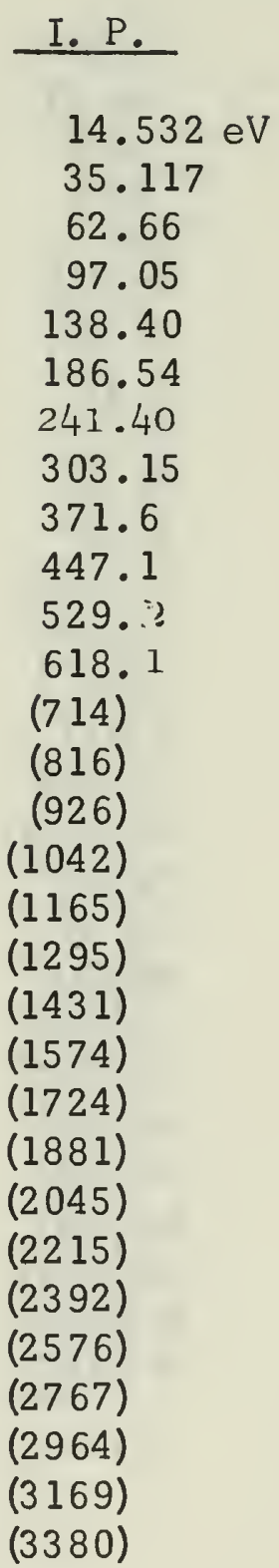


The non-relativistic extrapolated values are the CFF energies with a $\zeta^{2}$ correction subtracted.

Spectrum

O I

F II

Ne III

$\mathrm{Na}$ IV

$\mathrm{Mg} \mathrm{V}$

Al VI

Si VII

P VIII

$S$ IX

Cl X

Ar XI

K XII

Ca XIII

Sc XIV

Ti XV

$\checkmark$ XVI

Cr XVII

Mn XVIII

Fe XIX

Co XX

Ni XXI

$\mathrm{Cu}$ XXII

Zn XXIII

Ga XXIV

Ge XXV

As XXVI

Se XXVII

Br XXVIII

Kr XXIX

\section{Uncorrected}

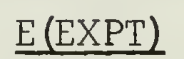

$13.618 \mathrm{eV}$

34.970

63.74

98.905

141.27

190.5

246.5

309.3

379.05

455.4

539.0

\begin{tabular}{c}
$E(\mathrm{CFF})$ \\
\hline $17.194 \mathrm{eV}$ \\
39.295 \\
68.459 \\
104.573 \\
147.58 \\
197.46 \\
254.20 \\
317.76 \\
388.16 \\
465.38 \\
549.43 \\
640.3 \\
738.0 \\
842.4 \\
953.7 \\
1072 \\
1197 \\
1328 \\
1467 \\
1612 \\
1764 \\
1923 \\
2089 \\
2262 \\
2441 \\
2627 \\
2820 \\
3020 \\
3227 \\
\end{tabular}

I. P.

$13.618 \mathrm{eV}$

34.970

63.74

98.905

141.27

190.5

246.5

309.3

379.05

455.4

538.8

(629)

(726)

(830)

(940)

(1058)

(1182)

(1313)

(145 1)

(1596)

(1747)

(1905)

(2070)

(2242)

(2421)

(2606)

(2798)

(2997

(3203) 
F Sequence:

The non-relativistic extrapolated values are the CFF energies with a $\zeta^{2}$ correction subtracted.

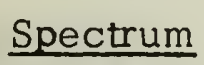

F I

$\mathrm{Ne}$ II

Na III

Mg IV

Al V

Si VI

P VII

$S$ VIII

Cl IX

Ar X

$\mathrm{K} X \mathrm{X}$

Ca XII

Sc XIII

Ti XIV

$\mathrm{V} X \mathrm{~V}$

Cr XVI

Mn XVII

Fe XVIII

Co XIX

$\mathrm{Ni} X X$

$\mathrm{Cu} X X I$

Zn XXII

Ga XXIII

Ge XXIV

As XXV

Se XXVI

Br XXVII

Kr XXVIII

\section{$\underline{E(E X P T)}$}

$17.422 \mathrm{eV}$

40.962

71.665

109.32

153.8

205.16

263.37

328.89

400.8

479.1

$655 ?$

Uncorrected

$\mathrm{E}(\mathrm{CFF})$

$19.864 \mathrm{eV}$

43.716

74.671

112.60

157.44

209.17

267.75

333.18

405.44

484.54

570.46

663.20

762.8

869.1

982.3

1102

1229

1362

1503

1650

1804

1965

2133

2307

2489

2677

2872

3073

\begin{tabular}{l} 
I.P. \\
\hline $17.422 \mathrm{eV}$ \\
40.962 \\
71.665 \\
109.32 \\
153.8 \\
205.16 \\
263.37 \\
328.89 \\
400.8 \\
479.6 \\
$(565)$ \\
$(657)$ \\
$(756)$ \\
$(862)$ \\
$(975)$ \\
$(1095)$ \\
$(1222)$ \\
$(1355)$ \\
$(1495)$ \\
$(1642)$ \\
$(1796)$ \\
$(1956)$ \\
$(2123)$ \\
$(2297)$ \\
$(2478)$ \\
$(2666)$ \\
$(2860)$ \\
$(3061)$ \\
\end{tabular}




\section{Ne Sequence:}

The non-relativistic extrapolated values (beyond Zn XXI) are the CFF energies with a $t^{2}$ correction added.

$\begin{array}{lc}\text { Spectrum } & \text { E(EXPT) } \\ \text { Ne I } & 21.564 \mathrm{eV} \\ \text { Na II } & 47.302 \\ \text { Mg III } & 80.137 \\ \text { Al IV } & 119.99 \\ \text { Si V } & 166.77 \\ \text { P VI } & 220.47 \\ \text { S VII } & 281.06 \\ \text { Cl VIII } & 348.4 \\ \text { Ar IX } & 422.8 \\ \text { K X } & 503.8 \\ \text { Ca XI } & 591.8 \\ \text { Sc XII } & 686.6 \\ \text { Ti XIII } & 788.4 \\ \text { V XIV } & 897.1 \\ \text { Cr XV } & 1013.0 \\ \text { Mn XVI } & 1135.9 \\ \text { Fe XVII } & 1266 \\ \text { Co XVIII } & 1403 \\ \text { Ni XIX } & 1546.9 \\ \text { Cu XX } & 1697.9 \\ \text { Zn XXI } & 1855.9 \\ \text { Ga XXII } & \\ \text { Ge XXIII } & \\ \text { As XXIV } & \\ \text { Se XXV } & \\ \text { Br XXVI } & \\ \text { Kr XXVII } & \end{array}$

$\begin{array}{cc}\begin{array}{c}\text { Uncorrected } \\ \text { E(CFF) }\end{array} & \text { I.P. } \\ 23.140 \mathrm{eV} & 21.564 \mathrm{eV} \\ 48.901 & 47.302 \\ 81.798 & 80.137 \\ 121.69 & 119.99 \\ 168.51 & 166.77 \\ 222.22 & 220.47 \\ 282.80 & 281.06 \\ 350.23 & 348.4 \\ 424.51 & 422.8 \\ 505.61 & 503.8 \\ 593.55 & 591.8 \\ 688.31 & 686.6 \\ 789.89 & 788.4 \\ 898.28 & 897.1 \\ 1013.5 & 1013.0 \\ 1135.5 & 1135.9 \\ 1264.4 & 1266.7 \\ 1400.0 & 1403.4 \\ 1542.5 & 1546.9 \\ 1691.7 & 1697.9 \\ 1847.8 & 1855.9 \\ 2011 & (2019) \\ 2180 & (2191) \\ 2356 & (2369) \\ 2540 & (2554) \\ 2730 & (2746) \\ 2927 & (2945)\end{array}$




\section{Na Sequence:}

We used the Edlén relativistic correction $\Delta_{R}=\left(2.012 \times 10^{-5}\right)(\zeta+5)^{4}$. The final extrapolation formula is (with $\zeta=Z-10$ ):

$$
\mathrm{T}=1.51719\left[\frac{\zeta^{3}+6.4304 \zeta^{2}+4.1064 \zeta-0.9786}{\zeta+2.1434}\right]+\left(2.012 \times 10^{-5}\right)(\zeta+5)^{4}
$$

Small corrections to the observed values were made at CI VII, K IX, Ni XVIII, $\mathrm{Cu} X I X, \mathrm{ZnXX}$. These are marked by underlining the corrected portion.

\begin{tabular}{|c|c|c|c|c|}
\hline Spectrum & $\underline{E(E X P T)}$ & $\begin{array}{l}\text { Uncorrected } \\
\mathrm{E}(\mathrm{CFF}) \\
\end{array}$ & $\Delta_{\mathrm{R}}$ & I. P. \\
\hline $\mathrm{Na} \mathrm{I}$ & $5.138 e$ & $4.955 \mathrm{eV}$ & $0.026 \mathrm{eV}$ & $5.138 \mathrm{eV}$ \\
\hline $\mathrm{Mg} \mathrm{II}$ & 15.035 & 14.733 & .048 & 15.035 \\
\hline Al III & 28.447 & 28.055 & .082 & 28.447 \\
\hline Si IV & 45.141 & 44.664 & .132 & 45.141 \\
\hline P V & 65.023 & 64.454 & .201 & 65.023 \\
\hline S VI & 88.051 & 87.373 & .295 & 88.051 \\
\hline $\mathrm{Cl}$ VII & 114.30 & 113.39 & .417 & 114.22 \\
\hline Ar VIII & 143.50 & 142.48 & .575 & $143 . \overline{50}$ \\
\hline $\mathrm{K} I \mathrm{X}$ & 175.782 & 174.65 & .773 & 175.84 \\
\hline Ca X & 211.269 & 209.86 & 1.018 & 211.269 \\
\hline Sc XI & 249.86 & 248.13 & 1.319 & 249.86 \\
\hline Ti XII & 291.545 & 289.44 & 1.680 & 291.545 \\
\hline V XIII & 336.377 & 333.79 & 2.112 & 336.377 \\
\hline Cr XIV & 384.295 & 381.18 & 2.622 & 384.295 \\
\hline $\mathrm{Mn} X \mathrm{~V}$ & 435.323 & 431.61 & 3.219 & 435.323 \\
\hline Fe XVI & 489.457 & 485.06 & 3.913 & 489.457 \\
\hline Co XVII & 546.816 & 541.56 & 4.713 & 546.816 \\
\hline Ni XVIII & 607.18 & 601.08 & 5.63 & $607 . \underline{30}$ \\
\hline $\mathrm{Cu}$ XIX & 670 & 663.63 & 6.68 & $670 . \overline{95}$ \\
\hline Zn XX & 737.9 & 729.22 & 7.86 & $737 . \overline{77}$ \\
\hline Ga XXI & & 797.83 & 9.19 & {$[808]$} \\
\hline Ge XXII & & 869.46 & 10.69 & {$[881]$} \\
\hline As XXIII & & 944.13 & 12.37 & [953] \\
\hline Se XXIV & & 1021.8 & 14.23 & [ 1037$]$ \\
\hline $\mathrm{Br} X X V$ & & 1102.5 & 16.30 & {$\left[\begin{array}{llll}1 & 1 & 2 & 0\end{array}\right]$} \\
\hline $\mathrm{Kr} X X V I$ & & 1186.3 & 18.58 & [1206] \\
\hline
\end{tabular}


Mg Sequence:

The non-relativistic extrapolated values (beyond Co XVI) are the CFF energies with a $\zeta^{2}$ correction added.

\begin{tabular}{|c|c|c|c|c|}
\hline Spectrum & $\underline{E(E X P T)}$ & $\begin{array}{l}\text { Uncorrected } \\
\mathrm{E}(\mathrm{CFF}) \\
\end{array}$ & I. P. & \\
\hline $\mathrm{Mg} \mathrm{I}$ & $7.646 \mathrm{ev}$ & $6.886 \mathrm{eV}$ & 7.646 & $\mathrm{ev}$ \\
\hline Al II & 18.828 & 17.749 & 18.828 & \\
\hline Si III & 33.492 & 32.161 & 33.492 & \\
\hline P IV & 51.367 & 49.881 & 51.505 & \\
\hline$S V$ & 72.49 & 70.800 & 72.49 & \\
\hline $\mathrm{Cl} \mathrm{VI}$ & 97 & 94.863 & 96.85 & \\
\hline Ar VII & 124.03 & 122.03 & 124.03 & \\
\hline K VIII & 155 & 152.29 & $15 \underline{4.8}$ & \\
\hline Ca IX & 188.0 & 185.62 & 188.5 & \\
\hline Sc $X$ & 225.5 & 222.01 & 225.5 & \\
\hline Ti XI & 265.6 & 261.46 & 265.6 & \\
\hline V XII & 308.0 & 303.96 & $308 . \underline{5}$ & \\
\hline Cr XIII & 354.8 & 349.49 & 354.8 & \\
\hline Mn XIV & 404.1 & 398.07 & 404.1 & \\
\hline $\mathrm{Fe} \mathrm{XV}$ & 456.7 & 449.69 & 456.7 & \\
\hline Co XVI & 512.4 & 504.34 & 512.4 & \\
\hline $\mathrm{Ni}$ XVII & & 562.03 & (57 1) & \\
\hline $\mathrm{Cu}$ XVIII & & 622.75 & (632) & \\
\hline Zn XIX & & 686.51 & (697) & \\
\hline Ga XX & & 753.29 & (765) & \\
\hline Ge XXI & & 823.10 & (836) & \\
\hline As XXII & & 895.94 & $(910)$ & \\
\hline Se XXIII & & 971.81 & (987) & \\
\hline $\mathrm{Br}$ XXIV & & 1050.7 & (1067) & \\
\hline $\mathrm{Kr} X \mathrm{XXV}$ & & 1132.6 & (1150) & \\
\hline
\end{tabular}




\section{Al Sequence:}

The non-relativistic extrapolated values (beyond Ar VI) are the CFF energies with a $\zeta^{2}$ correction added.

\begin{tabular}{lc} 
Spectrum & E(EXPT) \\
\cline { 2 - 2 } Al I & $5.986 \mathrm{eV}$ \\
Si II & 16.346 \\
P III & 30.163 \\
S IV & 47.304 \\
CI V & 67.8 \\
Ar VI & 91.32 \\
K VII & $118 ?$ \\
Ca VIII & $147 ?$ \\
Sc IX & $180 ?$ \\
Ti X & \\
V XI & \\
Cr XII & \\
Mn XIII & \\
Fe XIV & \\
Co XV & \\
Ni XVI & \\
Cu XVII & \\
Zn XVIII & \\
Ga XIX & \\
Ge XX & \\
As XXI & \\
Se XXII & \\
Br XXIII & \\
Kr XXIV &
\end{tabular}

\begin{tabular}{cc}
$\begin{array}{cc}\text { Uncorrected } \\
\text { E(CFF) }\end{array}$ & I.P. \\
\cline { 1 - 1 } $5.713 \mathrm{eV}$ & $5.986 \mathrm{eV}$ \\
15.492 & 16.346 \\
29.671 & 30.163 \\
46.711 & 47.304 \\
66.964 & 67.8 \\
90.372 & 91.32 \\
116.90 & $(120)$ \\
146.53 & $(150)$ \\
179.24 & $(183)$ \\
215.01 & $(220)$ \\
253.85 & $(259)$ \\
295.74 & $(301)$ \\
390.68 & $(347)$ \\
388.66 & $(395)$ \\
439.69 & $(446)$ \\
493.75 & $(500)$ \\
550.85 & $(556)$ \\
610.99 & $(616)$ \\
674.16 & $(679)$ \\
740.36 & $(744)$ \\
809.59 & $(812)$ \\
881.86 & $(883)$ \\
957.15 & $(957)$ \\
1035.5 & $(1034)$
\end{tabular}




\section{Si Sequence:}

The non-relativistic extrapolated values (beyond $\mathrm{K} V \mathrm{VI}$, but with observations at $\mathrm{Fe}$ XIII and $\mathrm{Ni} \mathrm{XV}$ ) are the CFF energies with a $\zeta^{2}$ correction subtracted.

\begin{tabular}{lc} 
Spectrum & E(EXPT) \\
\cline { 2 - 2 } Si I & \\
P II & $8.151 \mathrm{eV}$ \\
S III & 19.73 \\
Cl IV & 35.06 \\
Ar V & 53.46 \\
K VI & 75.02 \\
Ca VII & 99.74 \\
Sc VIII & $128 ?$ \\
Ti IX & $159 ?$ \\
V X & $193 ?$ \\
Cr XI & \\
Mn XII & \\
Fe XIII & \\
Co XIV & \\
Ni XV & $355 ?$ \\
Cu XVI & \\
Zn XVII & $455 ?$ \\
Ga XVIII & \\
Ge XIX & \\
As XX & \\
Se XXI & \\
Br XXII & \\
Kr XXIII &
\end{tabular}

\section{Uncorrected}

\begin{tabular}{|c|c|}
\hline$E(C F F)$ & I. P. \\
\hline $8.084 \mathrm{eV}$ & $8.151 \mathrm{eV}$ \\
\hline 19.591 & 19.73 \\
\hline 34.566 & 35.06 \\
\hline 52.840 & 53.46 \\
\hline 74.322 & 75.02 \\
\hline 98.96 & 99.74 \\
\hline 126.72 & $(127.3)$ \\
\hline 157.58 & $(157.9)$ \\
\hline 191.52 & $(191.4)$ \\
\hline 228.54 & (228) \\
\hline 268.61 & (267) \\
\hline 311.74 & $(310)$ \\
\hline 357.93 & $355 . \underline{5}$ \\
\hline 407.15 & $(404)$ \\
\hline 459.42 & 455.3 \\
\hline 514.74 & $(510)$ \\
\hline 573.08 & $(567)$ \\
\hline 634.47 & (627) \\
\hline 698.89 & $(690)$ \\
\hline 766.34 & $(756)$ \\
\hline 836.83 & (825) \\
\hline 910.34 & (897) \\
\hline 9 & $971)$ \\
\hline
\end{tabular}




\section{P Sequence:}

The non-relativistic extrapolated values are the uncorrected CFF energies, because of the small number of observations.

Spectrum

P I

S II

Cl III

Ar IV

$\mathrm{K} \mathrm{V}$

Ca VI

Sc VII

Ti VIII

$\mathrm{V}$ IX

Cr X

Mn XI

Fe XII

Co XIII

$\mathrm{Ni}$ XIV

$\mathrm{Cu} \mathrm{XV}$

Zn XVI

Ga XVII

Ge XVIII

As XIX

Se XX

Br XXI

Kr XXII

\section{$\underline{E(E X P T)}$}

$10.486 \mathrm{eV}$

23.411

39.914

58.81

\section{$\underline{E(C F F)}$}

$10.658 \mathrm{eV}$

23.449

39.677

59.19

81.9

108

137

169

204

242

283

328

375

426

479

536

596

658

724

793

864

939 


\section{S Sequence:}

The non-relativistic extrapolated values (beyond $\mathrm{Mn} \mathrm{X}$ ) are the CFF energies with a $\iota^{2}$ correction subtracted.

\section{Spectrum}

S I

Cl II

Ar III

K IV

$\mathrm{Ca} \mathrm{V}$

Sc VI

Ti VII

V VIII

Cr IX

$\mathrm{Mn} X$

$\mathrm{Fe} X I$

Co XII

$\mathrm{Ni}$ XIII

$\mathrm{Cu}$ XIV

Zn XV

Ga XVI

Ge XVII

As XVIII

Se XIX

Br XX

$\mathrm{Kr} X X I$

\section{E(EXPT)}

$10.360 \mathrm{eV}$

23.8

40.909

60.90

84.41

111.1

140.8

173.7

209.6

248

$290 ?$

Uncorrected

\begin{tabular}{l}
$E(\mathrm{CFF})$ \\
\hline $11.901 \mathrm{eV}$ \\
25.544 \\
42.643 \\
63.042 \\
86.66 \\
113.4 \\
143.3 \\
176.4 \\
212.5 \\
251.6 \\
293.9 \\
339 \\
388 \\
439 \\
493 \\
551 \\
611 \\
675 \\
742 \\
811 \\
884
\end{tabular}

\begin{tabular}{l} 
I.P. \\
\hline $10.360 \mathrm{eV}$ \\
23.8 \\
40.909 \\
60.90 \\
84.41 \\
111.1 \\
140.8 \\
173.7 \\
209.6 \\
248.3 \\
$(290.2)$ \\
$(335)$ \\
$(383)$ \\
$(434)$ \\
$(489)$ \\
$(546)$ \\
$(606)$ \\
$(669)$ \\
$(735)$ \\
$(804)$ \\
$(876)$
\end{tabular}




\section{Cl Sequence:}

The non-relativistic extrapolated values (beyond Co XI) are the uncorrected CFF energies. The corrections $\Delta_{\mathrm{CFF}}$ are quite small.

\begin{tabular}{|c|c|c|}
\hline Spectrum & $E(E X P T)$ & $\underline{E(\mathrm{CFF})}$ \\
\hline $\mathrm{Cl} \mathrm{I}$ & $12.967 \mathrm{eV}$ & $13.779 \mathrm{eV}$ \\
\hline Ar II & 27.629 & 28.444 \\
\hline $\mathrm{K}$ III & 46.5 & 46.56 \\
\hline $\mathrm{Ca}$ IV & 67.9 & 67.99 \\
\hline $\mathrm{Sc} \mathrm{V}$ & 92.5 & 92.64 \\
\hline Ti VI & 119.5 & 120.4 \\
\hline V VII & $151 . \underline{4}$ & 151.4 \\
\hline Cr VIII & $185 . \underline{4}$ & 185.4 \\
\hline Mn IX & $222 . \underline{6}$ & 222.6 \\
\hline Fe X & $262 . \overline{8}$ & 262.8 \\
\hline Co XI & $305 ?$ & 306.1 \\
\hline Ni XII & $350 ?$ & 352.5 \\
\hline $\mathrm{Cu}$ XIII & & 402 \\
\hline Zn XIV & & 454 \\
\hline Ga XV & & 510 \\
\hline Ge XVI & & 568 \\
\hline As XVII & & 630 \\
\hline Se XVIII & & 695 \\
\hline Br XIX & & 762 \\
\hline $\mathrm{Kr} X \mathrm{X}$ & & 833 \\
\hline
\end{tabular}


Ar Sequence:

The non-relativistic extrapolated values (beyond Fe IX) are the CFF energies with a $\zeta^{2}$ correction added.

\begin{tabular}{lccc} 
Spectrum & E(EXPT) & $\begin{array}{c}\text { Uncorrected } \\
\text { E(CFF) }\end{array}$ & I.P. \\
\cline { 3 - 3 } Ar I & $15.759 \mathrm{eV}$ & $16.082 \mathrm{eV}$ & $15.759 \mathrm{eV}$ \\
K II & 31.82 & 31.851 & 31.82 \\
Ca III & 50.908 & 51.069 & 50.908 \\
Sc IV & 73.9 & 73.592 & 73.9 \\
Ti V & 99.265 & 99.338 & 99.265 \\
V VI & 128.9 & 128.3 & 128.9 \\
Cr VII & 161.1 & 160.3 & 161.1 \\
Mn VIII & 196 & 195.4 & 196.1 \\
Fe IX & 235 & 233.8 & 234.7 \\
Co X & & 275 & $(276)$ \\
Ni XI & & 320 & $(321)$ \\
Cu XII & & 367 & $(369)$ \\
Zn XIII & & 418 & $(420)$ \\
Ga XIV & & 471 & $(474)$ \\
Ge XV & & 528 & $(531)$ \\
As XVI & & 588 & $(591)$ \\
Se XVII & & 650 & $(654)$ \\
Br XVIII & & 716 & $(720)$ \\
Kr XIX & & 785 & $(789)$
\end{tabular}




\section{K Sequence:}

The non-relativistic extrapolated values (beyond Fe VIII) are the uncorrected CFF energies. The corrections $\Delta_{\mathrm{CFF}}$ are quite small.

$\begin{array}{lcc}\text { Spectrum } & \text { E(EXPT) } & \text { E(CFF) } \\ \text { K I } & 4.340 \mathrm{eV} & 4.013 \mathrm{eV} \\ \text { Ca II } & 11.871 & 11.328 \\ \text { Sc III } & 24.76 & 24.61 \\ \text { Ti IV } & 43.25 & 43.35 \\ \text { V V } & 65.2 & 65.53 \\ \text { Cr VI } & 90.6 & 91.03 \\ \text { Mn VII } & 119.27 & 119.75 \\ \text { Fe VIII } & 151 . \underline{6} & 151.7 \\ \text { Co IX } & & 187 \\ \text { Ni X } & & 225 \\ \text { Cu XI } & & 266 \\ \text { Zn XII } & & 311 \\ \text { Ga XIII } & & 358 \\ \text { Ge XIV } & & 409 \\ \text { As XV } & & 462 \\ \text { Se XVI } & & 519 \\ \text { Br XVII } & & 579 \\ \text { Kr XVIII } & & 642\end{array}$




\section{Ca Sequence:}

The non-relativistic extrapolated values (beyond $\mathrm{Cr} V$ ) are the uncorrected CFF energies.

$\begin{array}{lll}\text { Spectrum } & \text { E(EXPT) } & \text { E(CFF) } \\ \text { Ca I } & 6.113 \mathrm{eV} & 5.320 \mathrm{eV} \\ \text { Sc II } & 12.80 & 12.225 \\ \text { Ti III } & 27.47 & 27.86 \\ \text { V IV } & 46.71 & 47.30 \\ \text { Cr V } & 73 ? & 70.2 \\ \text { Mn VI } & & 97 \\ \text { Fe VII } & & 126 \\ \text { Co VIII } & & 159 \\ \text { Ni IX } & & 195 \\ \text { Cu X } & & 234 \\ \text { Zn XI } & & 276 \\ \text { Ga XII } & & 321 \\ \text { Ge XIII } & & 369 \\ \text { As XIV } & & 421 \\ \text { Se XV } & & 475 \\ \text { Br XVI } & & 533 \\ \text { Kr XVII } & & 593\end{array}$

\section{Sc Sequence:}

The non-relativistic extrapolated values (beyond $\mathrm{Mn} V$ ) are the uncorrected CFF energies.

\begin{tabular}{lcc} 
Spectrum & E(EXPT) & $\underline{E(C F F)}$ \\
\cline { 2 - 3 } Sc I & $6.54 \mathrm{eV}$ & $5.72 \mathrm{eV}$ \\
Ti II & 13.58 & 13.00 \\
V III & 29.31 & 30.35 \\
Cr IV & 49.6 & 50.40 \\
Mn V & $76 ?$ & 74.0 \\
Fe VI & & 101 \\
Co VII & & 131 \\
Ni VIII & & 165 \\
Cu IX & & 201 \\
Zn X & & 241 \\
Ga XI & & 284 \\
Ge XII & 330 \\
As XIII & & 379 \\
Se XIV & & 431 \\
Br XV & & 486 \\
Kr XVI & & 545
\end{tabular}




\section{Ti-Ni Sequences:}

The non-relativistic extrapolated values are the uncorrected CFF energies.

Spectrum

Ti I

V II

Cr III

Mn IV

Fe V

Co VI

$\mathrm{Ni}$ VII

$\mathrm{Cu}$ VIII

Zn IX

Ga X

Ge XI

As XII

Se XIII

$\mathrm{Br}$ XIV

$\mathrm{Kr}$ XV
$\underline{E(E X P T)}$

$6.82 \mathrm{eV}$

14.65

30.96

53.2

53.2

77

105

136

170

207

248

291

338

388

441

497

\section{Sequence:}

Spectrum

V I

Cr II

Mn III

$\mathrm{Fe}$ IV

Co V

$\mathrm{Ni}$ VI

$\mathrm{Cu}$ VII

Zn VIII

Ga IX

Ge X

As XI

Se XII

Br XIII

Kr XIV
E(EXPT)

$6.74 \mathrm{eV}$

16.50

33.67
$\underline{E(C F F)}$

$6.27 \mathrm{eV}$

17.67

35.05

56

81

110

141

176

214

255

300

347

398

451 
Cr Sequence:

$\begin{array}{lrl}\text { Spectrum } & \underline{E(E X P T)} & \underline{E(C F F)} \\ \text { Cr I } & 6.765 \mathrm{eV} & 6.307 \mathrm{eV} \\ \text { Mn II } & 15.640 & 15.011 \\ \text { Fe III } & 30.651 & 35.833 \\ \text { Co IV } & & 57.52 \\ \text { Ni V } & & 82.8 \\ \text { Cu VI } & 111 \\ \text { Zn VII } & 144 \\ \text { Ga VIII } & 179 \\ \text { Ge IX } & & 217 \\ \text { As X } & & 259 \\ \text { Se XI } & & 304 \\ \text { Br XII } & & 352 \\ \text { Kr XIII } & & 403\end{array}$

Mn Sequence:

Spectrum

$\mathrm{Mn} \mathrm{I}$

$\mathrm{Fe}$ II

Co III

$\mathrm{Ni}$ IV

$\mathrm{Cu} \mathrm{V}$

Zn VI

Ga VII

Ge VIII

As IX

Se X

$\mathrm{Br} X I$

Kr XII
$\underline{E(E X P T)}$

$7.432 \mathrm{eV}$

16.182

33.50
$\underline{E(C F F)}$

$6.745 \mathrm{eV}$

15.35

37.17

59

85

114

147

183

222

264

310

358 
Fe Sequence:

\section{Spectrum}

Fe I

Co II

$\mathrm{Ni}$ III

$\mathrm{Cu}$ IV

Zn V

Ga VI

Ge VII

As VIII

Se IX

Br X

$\mathrm{Kr}$ XI
E(EXPT)

7. $90 \mathrm{eV}$

17.06

36.17

\section{$\underline{E(C F F)}$}

$7.02 \mathrm{eV}$

19.99

38.59

61

87

117

151

187

227

270

316

Co Sequence:

Spectrum

Co I

$\mathrm{Ni}$ II

$\mathrm{Cu}$ III

Zn IV

Ga V

Ge VI

As VII

Se VIII

$\mathrm{Br}$ IX

Kr X
$\underline{E(E X P T)}$

$7.86 \mathrm{eV}$

18.15

36.83

$\underline{E(E X P T)}$

$7.634 \mathrm{eV}$

20.291

39.722

64.2

93.4

127.5 ?

155 ?

193 ?
$\underline{E(C F F)}$

$$
7.28 \mathrm{eV}
$$

20.93

40.04

63

90

120

154

191

232

275

Ni I

Zn III

Ga IV

Ge V

As VI

Se VII

Br VIII

Kr IX

$\underline{E(C F F)}$

$7.517 \mathrm{eV}$

22.04

41.74

65.53

93.02

124.0

158

196

237 


\section{Cu Sequence:}

The non-relativistic extrapolated values (beyond Se VI) are the CFF energies with a $\zeta^{2}$ correction added.

Spectrum

$\mathrm{Cu} \mathrm{I}$

Zn II

Ga III

Ge IV

As $V$

Se VI

Br VII

Kr VIII

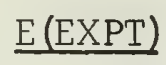

$7.726 \mathrm{eV}$

17.964

30.7

45.7

62.6

81.7

\section{Uncorrected}

\begin{tabular}{ll}
$E(C F F)$ & I.P. \\
\cline { 2 - 2 } $6.489 \mathrm{eV}$ & $7.726 \mathrm{eV}$ \\
16.64 & 17.964 \\
29.27 & 30.7 \\
44.07 & 45.7 \\
60.9 & 62.6 \\
79.7 & 81.7 \\
100 & $(103)$ \\
123 & $(125)$
\end{tabular}

\section{Zn-Ga Sequences:}

The few unobserved values shown are the result of a $\iota^{2}$ extrapolation of the experimental data.

\section{Ge - Kr Sequences:}

All the values shown are experimental.

$\mathrm{E}$ (EXPT) in eV for the $\mathrm{Zn}-\mathrm{Kr}$ Sequences:

\begin{tabular}{llllllll}
\multicolumn{9}{l}{ Spectrum Numiver } \\
& I & II & III & IV & V & VI & VII \\
& & & & & & & \\
$\mathrm{Zn}$ & 9.394 & 17.964 & 39.722 & $(63)$ & $(87)$ & $(114)$ & $(144)$ \\
$\mathrm{Ga}$ & 6.00 & 20.51 & 30.7 & 64.2 & $(90)$ & $(117)$ & $(147)$ \\
$\mathrm{Ge}$ & 7.88 & 15.93 & 34.22 & 45.7 & 93.4 & $(120)$ & $(151)$ \\
$\mathrm{As}$ & 9.81 & 20.2 & 28.3 & 50.1 & 62.6 & 127.5 & $(154)$ \\
$\mathrm{Se}$ & 9.75 & 21.5 & 32.0 & 42.9 & 73.1 & 81.7 & 155 \\
$\mathrm{Br}$ & 11.81 & 21.6 & 35.9 & {$[48]$} & {$[61]$} & {$[96]$} & $(103)$ \\
$\mathrm{Kr}$ & 13.999 & 24.57 & 36.95 & {$[53]$} & {$[67]$} & {$[82]$} & {$[123]$}
\end{tabular}




\section{REFERENCES:}

1. B. Edlén, Atomic Spectra, in Encyclopedia of Physics, Vol. 27 (Springer-Verlag, Berlin, 1964)

2. W. Lotz, J. Opt. Soc. Am. $\underline{57}, 873-78$ (1967)

3. W. Finkelnburg and W. Humbach, Naturwiss. 42, 35-37 (1955)

4. F. Herman and S. Skillman, Atomic Structure Calculations (Prentice-Hall, Inc., Englewood Cliffs, New Jersey, 1963)

5. C. F. Fischer, Can. J. Phys. 46,2336 (1968), and earlier references included there. We found that in several cases the starting values supplied by Mrs. Fischer would run the neutral atom, but not the entire isoelectronic sequence. We will be happy to supply starting values for $Z \leq 34$ which will run the sequences in the ground state configuration.

6. C. E. Moore, U. S. Nat. Bur. Stand., Circular 467, Atomic Energy Levels; Vol. I, 309 pp (1949), Vol. II, 227 pp (1952), Vol. III, $245 \mathrm{pp}$ (1958), (U. S. Government Printing Office, Washington, D. C.)

7. R. L. Kelly, NRL Report 6648, Atomic Emission Lines Below 2000 Angstroms, $354 \mathrm{pp}$ (1968), (U. S. Government Printing Office, Washington, D. C.) 
Dr. R. C. Elton

Naval Research Laboratory, Code 7720

Washington, D. C. 20390

Defense Documentation Center

Cameron Station

Arlington, Virginia 22314

Dr. B. C. Fawcett

Culham Laboratory

AERE-HARWELL

Berkshire, England

Dr. Bengt Edlén

Physics Department

Lund University

Lund, Sweden

Dr. Robert D. Cowan

University of California

Los Alamos Scientific Laboratory

Los Alamos, New Mexico

Dr. Kjell Bockasten

1

Lund Institute of Technology

Department of Physics

Lund, Sweden

Dr. H. E. Hinteregger

Upper Atmosphere Physics Laboratory

Headquarters Air Force Cambridge Research

Laurance G. Hanscom Field

Bedford, Massachusetts 01731

Dr. Boris Ragent (230-1)

Ames Research Center

National Aeronautics and Space Administration

Moffett Field, California

Dr. C. E. Moore Sitterley

National Bureau of Standards

Washington, D. C. 20234

Dr. Victor Kaufman

National Bureau of Standards

Washington, D. C. 20234 
Dr. Lucy Hagan

Atomics and Molecular Physics Division

National Bureau of Standards

Washington, D. C. 20234

Dr. Thomas A. Carlson

Physics Division

Oak Ridge National Laboratory

Oak Ridge, Tennessee 37831

Library

Naval Postgraduate School

Monterey, California

Central Files

Naval Postgraduate School

Monterey, California

Dean Carl E. Menneken

Research Administration

Naval Postgraduate School

Monterey, California

Professor R. L. Kelly

Physics Department

Naval Postgraduate School

Monterey, California

Professor D. E. Harrison, Jr.

Physics Department

Naval Postgraduate School

Monterey, California 


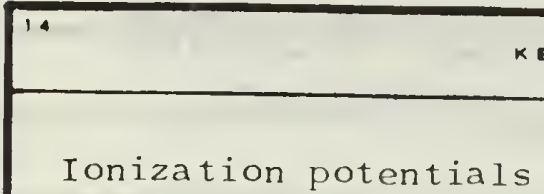

Multiply-charged ions

Is oelectronic sequence

Hartree-Fock calculations 


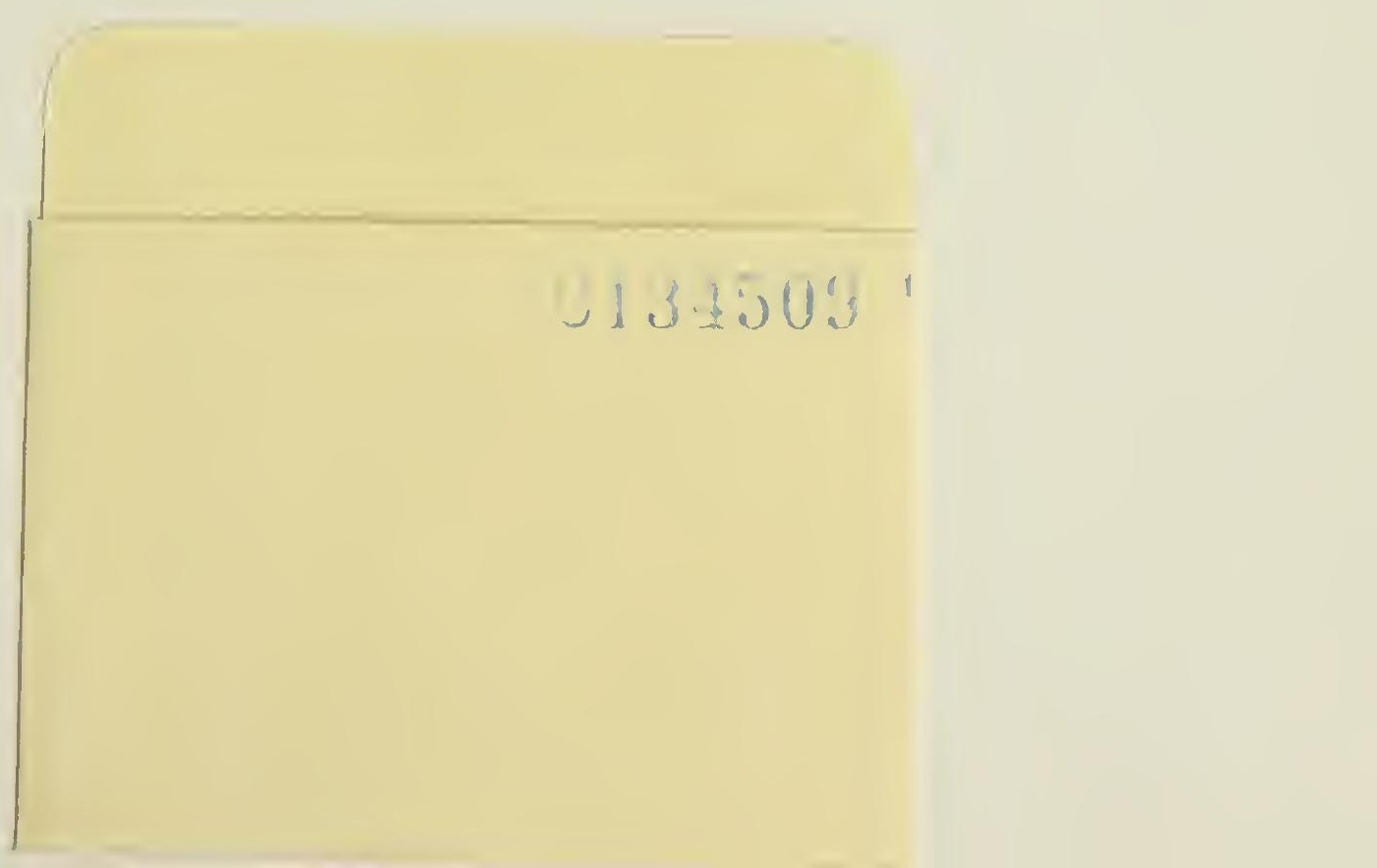


OUDLEY KNOX LIBRARY - RESEARCH REPORTS
IHIIIN)

56853010582471 